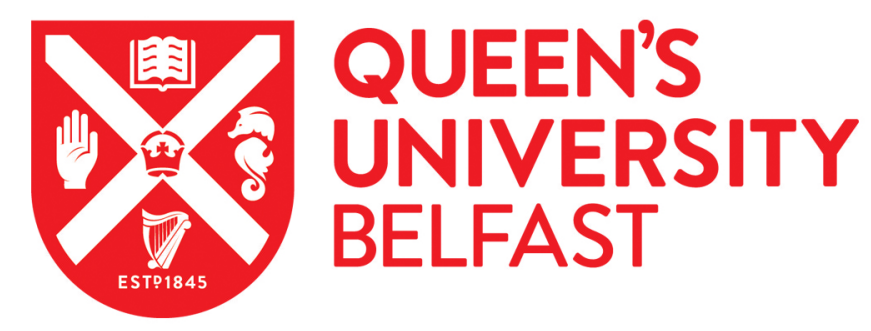

\title{
Introducing the DM-P approach for analysing the performances of real-time clinical decision support systems
}

Novakovic, A., \& Marshall, A. H. (2020). Introducing the DM-P approach for analysing the performances of realtime clinical decision support systems. Knowledge-Based Systems, 198, [105877].

https://doi.org/10.1016/j.knosys.2020.105877

\section{Published in:}

Knowledge-Based Systems

\section{Document Version:}

Version created as part of publication process; publisher's layout; not normally made publicly available

Queen's University Belfast - Research Portal:

Link to publication record in Queen's University Belfast Research Portal

\section{Publisher rights}

Copyright 2020 Elsevier.

This manuscript is distributed under a Creative Commons Attribution-NonCommercial-NoDerivs License

(https://creativecommons.org/licenses/by-nc-nd/4.0/), which permits distribution and reproduction for non-commercial purposes, provided the author and source are cited.

\section{General rights}

Copyright for the publications made accessible via the Queen's University Belfast Research Portal is retained by the author(s) and / or other copyright owners and it is a condition of accessing these publications that users recognise and abide by the legal requirements associated with these rights.

Take down policy

The Research Portal is Queen's institutional repository that provides access to Queen's research output. Every effort has been made to ensure that content in the Research Portal does not infringe any person's rights, or applicable UK laws. If you discover content in the Research Portal that you believe breaches copyright or violates any law, please contact openaccess@qub.ac.uk. 


\section{Journal Pre-proof}

Introducing the DM-P approach for analysing the performances of real-time clinical decision support systems

Aleksandar Novakovic, Adele H. Marshall

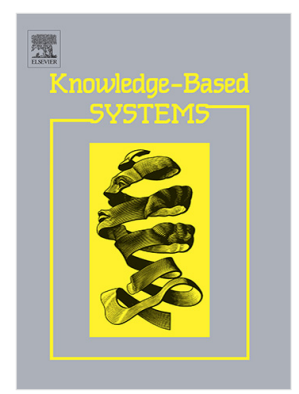

PII:

S0950-7051(20)30232-X

DOI: $\quad$ https://doi.org/10.1016/j.knosys.2020.105877

Reference: KNOSYS 105877

To appear in: Knowledge-Based Systems

Received date: 8 August 2019

Revised date: 29 February 2020

Accepted date : 3 April 2020

Please cite this article as: A. Novakovic and A.H. Marshall, Introducing the DM-P approach for analysing the performances of real-time clinical decision support systems, Knowledge-Based Systems (2020), doi: https://doi.org/10.1016/j.knosys.2020.105877.

This is a PDF file of an article that has undergone enhancements after acceptance, such as the addition of a cover page and metadata, and formatting for readability, but it is not yet the definitive version of record. This version will undergo additional copyediting, typesetting and review before it is published in its final form, but we are providing this version to give early visibility of the article. Please note that, during the production process, errors may be discovered which could affect the content, and all legal disclaimers that apply to the journal pertain.

(C) 2020 Published by Elsevier B.V. 


\section{Introducing the DM-P approach for analysing the performances of real-time clinical}

\section{decision support systems}

Aleksandar Novakovic ${ }^{*}, 1$ and Adele H Marshall ${ }^{*}, 1$

* Sharing first authorship

1. School of Mathematics and Physics, Queen's University Belfast, University Road, Belfast Northern Ireland BT7 1NN, United Kingdom

\section{Abstract}

This paper introduces the DM-P approach for assessing the performance of the alerts produced from a clinical decisions support system when there is no information to indicate a genuine observation and one that is not. The DM-P approach allows the representation of the p-charts, normally used in a statistical process control chart setting, as a defects map which is ordered according to the occurrence of similar behaviour as determined using agglomerative hierarchical clustering. The effectiveness of this new approach is demonstrated using real life data from the VILIAlert clinical decisions support system deployed in real-time in an intensive care unit to monitor patients on the ventilator and used to trigger alerts to clinicians when a patient is at risk of receiving injurious mechanical ventilation. The VILIAlert data was successfully used as a case study for the DM-P approach proving both the usefulness of the technique and its ability to identify the genuine cases of patients at risk. Such an approach also has scale up quality being designed in such a way to be able to deal with large volumes of data and can be extended to include any form of statistical process control chart not just p-charts.

Keywords: performance analytics, clinical decision support, real-time alert, defects map, pchart, statistical process control 


\section{Introduction}

Clinicians working in Intensive Care Units (ICUs), are very often required to deliver between 80 and 200 evidence-based interventions on a daily basis, per patient [1], with one of these interventions being to ensure adequate quality of mechanical ventilation [2]. In spite of the fact that it is one of the crucial life supporting facilities [3], mechanical ventilation is an intrusive procedure which can lead to permanent damage to the lungs (i.e. ventilator induced lung injury [VILI]) if not conducted properly [4]. Numerous studies have demonstrated that VILI is a preventable condition if the lung protective ventilation (LPV) strategy is applied, by which patients are ventilated with low tidal volumes (TVs), usually $<8 \mathrm{ml} / \mathrm{kg}$ of ideal body weight (IBW) [5] [6] [7]. However, current evidence shows that the LPV strategy adoption has been inconsistent [8] [9], with less than $50 \%$ of all patients receiving low tidal volume in the ICU settings [10] [11] [12]. Barriers to adopting best practices are multifactorial and some possible explanation include, but are not limited to, clinicians having: competing work demands, using actual patients' body weight instead of IBW for calculating TVs in $\mathrm{ml} / \mathrm{kg}$ units, or the lack of knowledge [2] [3].

In order to elevate the use of the best LPV practice, we designed VILIAlert - a novel Healthcare 4.0 compliant real-time clinical decision support system (CDSS) with the purpose to continuously monitor patients' TVs and alert clinicians if it predicts that configured ventilation settings can cause VILI [13]. VILIAlert was deployed to the Regional ICU ward at the Royal Victoria Hospital Belfast, where it was operating for nearly 2 years. The ward has a maximum capacity of 20 patients, with the platform showing promise to be scaled out to a hospital wide level [14].

Bearing in mind that clinically insignificant alerts can induce alert fatigue [15], the main hindrance in designing smart alerting CDSSs such as that presented in this paper, is to make them robust enough so the clinicians are alerted only when there are genuine alerts. However, distinguishing a difference between these two types of alerts is very difficult and requires assistance of the clinical experts. If we take into account clinicians' competing work 
demands, it is unreasonable to expect their feedback on authenticity to be provided immediately upon the alert generation. Therefore, accuracy assessment is usually performed retrospectively, very often at the end of the study, with each alert being manually inspected and a decision made as to whether they were genuine or not. In our opinion, this approach to manual accuracy assessment relying on clinical support, has two main issues: (i) it is a very laborious and time consuming task, especially if taken into account that a large number of alerts can be accumulated over time for inspection (depending on the CDSS configuration settings, quality of alerting algorithm, number of patients involved in the study, etc.) and, (ii) the development of novel algorithms is constrained by the lack of adequate metrics and tools which could be used in the evaluation of their performance, as well as lead towards the minimisation of dependence on the clinical assistance.

To facilitate these issues and minimise the need for the clinician's input in evaluating the performance of the real-time smart alerting CDSS, we propose the DM-P approach. This novel hybrid approach, is based on the combination of p-charts, which are the type of statistical process control (SPC) charts used in quality control of dichotomous processes, and the visualisation technique also introduced in this paper that we named defects map (DM). Our main motivation behind the DM-P approach comes from the fact that we received no clinical feedback on the triggered alerts as to whether they were genuine or not, and hence standard evaluation metrics such as accuracy, area under curve (AUC) or precision/recall could not be used for assessing VILIAlert's performance.

To mitigate this problem, we hypothesised that if the alert was generated because the TV readings violated the $8 \mathrm{ml} / \mathrm{kg}$ threshold, then it is expected that this threshold is going to be continuously violated for some considerable time in the future. This hypothesis was inspired by the work of Bordeaux et al. [2] in which they created a nudged based intervention to increase compliance with LPV in the ICU. In their study, they demonstrated that if the alert was triggered for the values of tidal volume $>8 \mathrm{mg} / \mathrm{kg}$, these values continue to remain above this threshold until the clinical intervention is performed. 
DM-P is a performance evaluation metric and as such intended for the analysis of performance of real-time Healthcare 4.0 smart alerting systems to inform clinical decision making. We demonstrated the effectiveness of the DM-P approach using the data obtained during the period of operation of the VILIAlert system. It can therefore be applied within an integrated module for any system or be used as an independent module in conjunction with other systems.

This paper has the following structure. In Section 2 we provide an architectural overview of the VILIAlert system, as well as provide the theoretical foundation behind DM-P approach for analysing the performance of unlabelled datasets. In Section 3 we provide a demographics overview of the patients involved in the study and present the accuracy assessment results of the VILIAlert system obtained through the use of the DM-P approach. In Section 4 we provide further discussion of our results and perform a comparison of related work from the literature. Finally, in Section 5 we provide concluding remarks as well as the direction of the future research.

\section{Methods}

\subsection{The VILIAlert system}

Mechanical ventilators (MVs) are the instruments used for providing breathing support, and in ICUs are used for patients who develop respiratory failure [5]. MVs, like many other organ support machines, are usually designed to produce patients' readings in the form compatible with the Health Level 7 (HL7) protocol which defines a set of guidelines for exchange, sharing and retrieval of electronic health information [16] [17]. These readings are commonly transferred to the Electronic Medical Records (EMRs), massive repositories designed to store large quantities of patients' data, so that the patient's changing health condition can be tracked and analysed over time [18] [19].

The VILIAlert system was designed to run in parallel with EMR to continuously monitor respiratory readings, to alert clinicians if it predicts that configured MV settings could lead to VILI in ventilated patient(s). The system became active in November 2015 in the Royal 
Victoria Hospital Belfast, and in the first couple of months it has undergone several tuning phases in which we altered data collection and alerting processes. Performance analysis of the VILIAlert system presented in this paper, is based on the dataset obtained in the period between 31.08.2016-26.10.2017, which corresponds to the dates when the system became stable and stopped being in use, respectively. The VILIAlert system works as follows.

Let's assume that $\mathbb{P}=\{N \mid N \in \mathbb{N}\}$ is the set of all ventilated patients, and that each patient $P \in \mathbb{P}$, was ventilated for the maximum time denoted by $M^{(P)}$ minutes. The VILIAlert system receives one tidal volume reading $T V_{m}^{(P)}$ in $\mathrm{mL} / \mathrm{inspiration}$ units, per patient $P$, per minute $m$, where $1 \leq m \leq M^{(P)}$, and stores these in a database. For each ventilated patient $P$, at fifteen minute intervals $i, 1 \leq i \leq\left\lceil\frac{M^{(P)}}{15}\right\rceil$, an analytics kernel computes the average tidal volume in $\mathrm{mL} / \mathrm{kg}$ units, the $\operatorname{TVMLKG_{i}^{(P)}}$ metric, using the following formula:

$$
T V M L K G_{i}^{(P)}=\frac{1}{15 \cdot I B W^{(P)}} \sum_{m=15 \cdot i-14}^{15 i} T V_{m}^{(P)}
$$

where $I B W^{(P)}$ denotes the ideal body weight of patient $P$ in kilograms which depends on their height $\left(H t_{\text {inches }}^{(P)}\right)$ and gender $\left(P_{\text {gender }}\right)$ obtained using the Devine formula [20]:

$$
I B W^{(P)}= \begin{cases}50.0+2.3 \cdot\left(H t_{\text {inches }}^{(P)}-60\right), & P_{\text {gender }}=\text { "Male" } \\ 45.5+2.3 \cdot\left(H t_{\text {inches }}^{(P)}-60\right), & P_{\text {gender }}=\text { "Female" }\end{cases}
$$

If this computed metric TVMLKG $G_{i}^{(P)}$ exceeds the threshold of $8 \mathrm{~mL} / \mathrm{kg}$ for four consecutive intervals, then an alert is generated for patient $P$, and the notification containing relevant patient information is sent as an SMS to the clinical staff's mobile phones. Upon receiving notification, it is the responsibility of the clinician to intervene in a timely manner to ensure that the optimal quality of mechanical ventilation care is delivered to the patient. In order to avoid excessive notification messages to clinical staff, the VILIAlert system is configured not to generate any new alert notification for the patient $P$ in a duration of 12 hours upon triggering the last alert. A schematic overview of the VILIAlert system, as well as a short summary of the analytical kernel's algorithm is shown in Figure 1. 


\section{Figure 1.}

An architectural overview of the VILIAlert system.

\subsection{Analysing VILIAlert's Performance using DM-P approach}

The analysis begins at the timestamp $t_{A}$ where the alert $A$ has been triggered at time $t$ for the patient $P$. At every fifteen minute interval, the metric $T V M L K G_{i}^{(P)}$ is computed and analysed over a six hour period of time since the trigger of the alarm (the period of time that covers 24 consecutive fifteen minute intervals since the alert generation), where $\left(t_{A}+1\right) \leq i \leq\left(t_{A}+\right.$ $24)$ and $\left(t_{A}+24\right) \leq\left\lceil\frac{M^{(P)}}{15}\right\rceil$. Let's denote this period of 24 consecutive fifteen minute intervals $i$ as the APA Time-Window (Analysed Post Alert Time-Window). Our key assumption is that if the alert has been triggered at the timestamp $t_{A}$ then the future $T V M L K G_{i}^{(P)}$ values will remain above the $8 \mathrm{~mL} / \mathrm{kg}$ threshold as well. In the case that this does not happen, and there is an interval $i$ for which computed metric $T V M L K G_{i}^{(P)}$ does not breach this threshold (i.e. $\left.T V M L K G_{i}^{(P)} \leq 8\right)$, we consider such an interval as "defective".

Ideally, if the alert is genuine we would expect no defective intervals (DIs) at all during the APA time window. However, this might not always be the case bearing in mind that the length of the APA time window is six hours (i.e. 24 fifteen minute intervals) during which time many external factors may have an impact on the overall ventilation process and thus result in obtaining $T V M L K G_{i}^{(P)}$ readings below the threshold. Due to the fact that the analysed dataset did not include any information on whether the clinical staff intervened upon receiving the VILIAlert notification or not, by converting a set of instantaneous $T V M L K G_{i}^{(P)}$ readings to a set of binary data, the accuracy of the VILIAlert system can be assessed through the analysis of emerging patterns during the APA time window. For this, we propose the use of the well established quality control approach of p-charts in combination with our new "defects map" visualisation technique, described in the text to follow.

\subsubsection{Using P-charts for Accuracy Assessment of the VILIAlert System}


P-charts are a type of statistical process control (SPC) charts used for analysing and monitoring dichotomous processes, and in recent years they are being frequently used in healthcare for measuring clinical performance and delivering continuous quality improvement [21] [22] [23] [24]. These charts resemble traditional time series plots accompanied by three additional horizontal lines - centreline $(C L)$ and two control limits, used for identifying central tendency and the natural variability of the monitored process respectively [25].

In this study, we use the p-chart for assessing the accuracy and stability of the VILIALert system, and construct it so that each data point (event) represents a proportion of defective intervals during the APA time window per triggered alert. The upper control limit (UCL) is constructed using 3 binomial-based standard deviations above the centreline, while on the other hand there was no lower control limit (LCL) as $0 \%$ defective intervals is preferable. This way of defining CLs is a common approach in practice. For instance, Padula et al. [26] in their study on systematic improvement of the prevention of hospital-acquired pressure ulcers, defined the CLs of their p-chart in the same manner as defined in our study.

The alert is defined as 'genuine' if the proportion of its defective intervals during the APA time window is within the control limits of the p-chart, and the calculated overall accuracy of the VILIAlert system is the ratio between the number of genuine and the total number of alerts. This is in compliance with one of the Health Care Rules (HCR) which states that any data point residing outside the control limits might be due to some special cause and hence should be further investigated [27]. Bearing in mind that events in the p-chart are without inherent natural ordering, it is important to note that other HCR that take into account run length and trend of the data points for detecting special cause variation were not applicable in the VILIAlert scenario.

\subsubsection{Introducing the Defects Map (DM) approach for investigating special cause of variation} in the p-charts

Defects Maps (DMs) are inspired by matrix plots for exploring incomplete datasets [28]. Similar to the matrix plot which shows the exact position of the missing values in a dataset, a 
DM displays the position of defective intervals relative to the timestamp of their occurrence during the APA time windows. In order to achieve this, all cells in a data matrix are visualised as rectangles and a bi-colour scheme is used to make a differentiation between defective and non-defective intervals.

However, what makes the matrix plots visualisation approach unsuitable for use in this study, is the fact that with the increase of the size of the dataset, it becomes very difficult to extract meaningful visual patterns which would help differentiate the genuine from the false alerts. A particularly challenging task is the identification of the genuine alerts which, due to the influence of external factors, might have an unusually high number of defective intervals recorded during the APA time window and hence may get misclassified.

To address these issues, before the visualisation step, the DM relies on agglomerative hierarchical clustering $(\mathrm{AHC})$ to rearrange the APA time window observations in a manner in which those with similar defective intervals' occurrence patterns appear next to each other in a data matrix. At the initial step, the AHC algorithm treats each APA time window observation as a separate atomic cluster, and based on a predefined linkage criterion and the dissimilarity metric, starts merging atomic clusters into larger clusters until all observations belong to a single cluster [29]. The strengths of the AHC approach is that the overall clustering process can be visualised as a tree-like structure called a dendogram which can be used for exploring cluster structure, as well as for determining the optimal number of clusters [30]. As a dissimilarity metric we used Euclidean distance, while for the linkage criteria we chose Ward's Minimum Variance method [31] which in comparison to other frequently used linkage criteria (such as average, complete or single linkage) has a better clustering accuracy [32] and lower computational costs [33]. A particularly important characteristic that differentiates Ward's from the other linkage methods is that it aims to create clusters with maximum internal cohesion (in a way that the criterion minimises withincluster distances) and maximum external separation (in a way that the criterion maximises between-cluster distances) [34]. This ensures the three scenarios of APA time window are clearly distinguishable in a DM by grouping APA time windows together that belong to the 1) 
false alerts, 2) genuine alerts, and 3) the genuine alerts where there was an influence of external factors during the APA time window.

To illustrate how the DM is constructed, we have provided a theoretical example of a data matrix similar to the one used in our analysis, illustrated in Figure 2 a). This dataset consists of five rows representing the APA time windows (one APA time window per row), each split into 24 columns representing consecutive fifteen-minute intervals. In order to differentiate non-defective intervals out of the ones that are defective, each interval $i$ (i.e. cell) in the data matrix is denoted with either 0 or 1 depending on whether the calculated $T V M L K G_{i}^{(P)}$ metric for that particular interval exceeds $8 \mathrm{~mL} / \mathrm{kg}$ threshold or not, respectively. Figure $2 \mathrm{~b}$ ) illustrates how this data matrix can be represented using the matrix plot visualisation technique, with the grey and black rectangles denoting non-defective and defective intervals, respectively. The DM shown in the figure $2 \mathrm{c}$ ) is constructed such that the APA time windows are firstly shuffled in the data matrix using the AHC algorithm so those with similar defective interval patterns appear next to each other. This is followed by the visualisation step in which positions of both defective and non-defective intervals are visualised using the same bicolour scheme as the previously described matrix plots.

In the example provided (Figure $2 a) \& b$ )), we presented four patterns occurring most of the time in the analysed dataset, each having a different interpretation. The first two patterns illustrated by the alerts $A=\{1\}$ and $A=\{2,5\}$ indicate false positive (FP) and genuine alerts respectively. Alert $A=\{1\}$ is classified as FP as our investigations of collected TVMLKG readings revealed that the alerts that exhibited this pattern were triggered due to spurious ventilatory readings, because of which their APA time window consisted of defective intervals only (indicated by all black cells in the Figure $2 \mathrm{~b}$ ). However, this was not the case with the alerts $A=\{2,5\}$, their APA time windows consisted of non-defective intervals only (indicated by all grey cells in the Figure $2 \mathrm{~b}$ ) because of which they were classified as genuine. 
Although it might not be as obvious at first glance, there are two remaining patterns for alerts $A=\{3\}$, and $A=\{4\}$ that should be considered. These can also indicate genuine cases of alerts. Alert 4 has a APA time window which consists of both defective and non-defective alerts where the first 17 consecutive intervals, corresponding to the first 255 minutes of ventilation time from triggering the alert, have calculated $T V M L K G_{i}^{(P)}$ metrics are above the threshold, and then suddenly from the interval $i=18$, this metric drops and remains below the threshold until the end of monitored time. This could be the result of clinical intervention of some kind (such as the change of the ventilation mode), in the period between the $255^{\text {th }}$ and $270^{\text {th }}$ minute since the alert was triggered, resulting in stabilised health readings, i.e. lower TVMLKG values.

Alert 3 does not follow the same pattern where it is apparent that there are fluctuations occurring above and below the $8 \mathrm{~mL} / \mathrm{kg}$ threshold. This could be as a result of a patient's conditions fluctuating in and out of control and again could include external influences where there has been clinical intervention. This is more evident when considering DM (Figure 2c) in which alerts with similar Defective Interval (DI) occurrence patterns are grouped together. In the next section we will describe how the p-charts and DM can be used in conjunction with one another for the purpose of analysing the performance of real-time alerting systems when there is no prior information which could be used to differentiate genuine alerts from ones that are not.

\section{Figure 2.}

An illustration on how the DM is constructed: a) Theoretical representation of the data-matrix similar to the one used in our analysis. Symbols 1 and 0 denote defective and non-defective intervals, respectively; b) Visualisation of the previously introduced data-matrix using matrix plot visualisation technique. Defective and non-defective intervals are represented as the black and grey cells respectively; c) a DM representation of the data-matrix in which the APA time window observations are arranged based on defective intervals occurrence patterns using the AHC algorithm (Ward's method with Euclidean distances).

\subsubsection{The DM-P approach for analysing authenticity of triggered alerts}


The DM-P approach for the validity identification of triggered alerts is a combination of two visual techniques: DM (described in the previous section) and p-charts (described in 2.2.1). It works as follows. Step 1 uses the APA data matrix (M) to construct the DM by applying the $\mathrm{AHC}$ algorithm to rearrange the APA time window observations in a manner in which those with similar DI occurrence patterns appear next to each other in a data matrix. Let's denote this $\mathrm{AHC}$ rearranged data matrix as M-DM (Step 2). In Step 3, we use a dendogram to determine the optimal number of clusters and identify their boundaries (CBs) in M-DM, so we can perform better context separation in order to make the interpretation of results easier (these CBs will be used later on in the Steps $5 \& 6$ ).

Although, in section 2.2.1 we proposed constructing p-charts using the proportions of DIs per APA time window observation, in the case of large and unstructured datasets, the p-charts have a tendency to become unreadable (see Fig. 3). Therefore, to overcome this issue the p-chart $(P)$ is constructed using the M-DM dataset instead, which then enforces the order of observations in $\mathrm{P}$ to be the same as in DM (Step 4). This not only makes the p-chart more readable and interpretable (which is obvious by making a comparison between the Fig. 3 and Fig. 4 b)), but it also allows its use in conjunction with DM thus enabling effective cross analysis possible between the two. This is a very important property because for each APA time window observation in $\mathrm{P}$ which exhibits special cause variation (i.e. contains unusually high number of DIs) we can investigate its exact DI occurrence pattern using DM and therefore differentiate genuine from the false alerts. In order to make this investigation easier, in the last two steps (Steps 5 and 6) we use horizontal and vertical dashed lines to update the DM and $\mathrm{P}$ charts and mark CBs location (obtained from the Step 3). Finally, by combining these two charts we get to the DM-P chart which resembles very much the one showed in Fig. 4. The pseudo-code of the DM-P approach is given in Alg 1.

Algorithm 1. The pseudo-code of DM-P visualisation algorithm Input: APA data matrix $\boldsymbol{M}$ in which the observations are arranged in chronological order Output: $\mathbf{D M - P}$ chart 
1. Let $\boldsymbol{D M}$ be the defect map constructed using $\boldsymbol{M}$

2. Let $\boldsymbol{M}-\mathbf{D} \boldsymbol{M}$ be the APA data matrix corresponding to the $\boldsymbol{D M}$ layout in which the observations are ordered based on their Dls occurrence pattern using $\mathrm{AHC}$

3. Let $\mathbf{C B s}$ be identified cluster boundaries in the $\mathbf{D M}$ using the dendogram approach

4. Let $\boldsymbol{P}$ be the p-chart constructed using the proportion of $D / \mathbf{s}$ in the $M-D M$ dataset

5. Update $\mathbf{D M}$ such that the $\mathbf{C B s}$ are denoted with the horizontal dashed lines

6. Update $\boldsymbol{P}$ such that the $\boldsymbol{C B s}$ are denoted with vertical dashed lines

7. Return the combined $\boldsymbol{D M}$ and $\boldsymbol{P}$ chart

\section{Results}

In this study we used routinely collected anonymised tidal volume along with gender and height data that has been captured during the period of operation of the VILIAlert system, for mechanically ventilated patients in both modes of ventilation ("control" and "support"). The Mann-Whitney-Wilcoxon and chi-square test of independence $\left(\chi^{2}\right)$ were used for investigating associations between gathered quantitative and qualitative variables and triggering the alert. Pearson's $r(r)$ computed from the standard normal deviate $Z$ [35], and Cramér's $V(V)$ coefficients were used as the effect size estimates of these two tests, and for their interpretation we used the guidelines proposed by Akoglu [36] and Chan [37], respectively. All analyses were conducted using $\mathrm{R}$ version 3.5.0 [38].

\subsection{The overview of ventilated patients' demographics}

During the period of 14 months of operation of the VILIAlert system, 875 patients received mechanical ventilation, and a total of 1450 alerts were triggered. Of all ventilated patients, $338(38.63 \%)$ were females, of whom $262(77.51 \%)$ triggered at least one alert. On the other hand, of all ventilated male patients 298 (55.49\%) invoked triggering an alert at least once. We identified a strong association between the gender and alert invocation $\left(\chi_{1}^{2}=42.71 ; p=\right.$ $0 ; V=.22$ ), indicating that upon starting mechanical ventilation female patients have a higher chance of triggering the alert. We also identified a fair association between the height and 
triggering the alert, implying that the shorter patients have more chance of generating an alert $(W=119960 ; p<.0001 ; r=.3)$. The median (MD) height of the patients who triggered the alert was $165 \mathrm{~cm}(I Q R=14.8 \mathrm{~cm})$, compared to $170 \mathrm{~cm}(I Q R=15.0 \mathrm{~cm})$ of those who have not triggered an alert. A similar analogy applies for IBW as the height measurements are used in their calculation (

Table 1).

Table 1: Comparison of ventilated patients' demographics; triggered vs. not triggered alerts

\subsection{VILIAlert's Accuracy assessment using DM-P approach}

Figure 3 shows a p-chart of the proportion of defective intervals relative to the APA time window of alerts aligned in chronological order. The overall mean percentage defective intervals (CL) was $25.8 \%$, which translates to 6.19 defective intervals per APA time window on average, with a UCL of $52.6 \%$ (denoted as the solid and dotted black horizontal lines in the graph, respectively). This indicates that the alert is genuine if the number of defective intervals does not exceed 12 (out of 24) per APA time window, and implies the overall VILIAlert's accuracy of $76.7 \%$ (1112/1450 APA time window observations within CLs). A special cause variation was detected in $338 / 1450$ cases, represented as the red dots in a graph which exceed the UCL.

\section{Figure 3.}

P-chart displaying the proportion of defective intervals relative to the APA time window observations which are arranged in chronological order. Upper control limit and centreline are denoted with dotted and solid black horizontal lines. Lower control limit is not set, as the $0 \%$ of defective intervals per APA time window is preferred. Light blue and red dots, depicts APA time window observations that indicate that the corresponding alert is either genuine or requires further investigation so that the authenticity of observations can be determined. 
A DM used to validate the accuracy of the VILIAlert system and inspect the roots of special cause variations, is shown in Figure 4 a). Using the dendogram approach we have identified 3 distinct clusters, containing $961\left(C_{1}\right), 212\left(C_{2}\right)$ and $277\left(C_{3}\right)$ APA time window observations each. As the DM arranges APA time window observations relative to their defective intervals' occurrence patterns, the p-chart is updated so that each APA time window observation appears in the same order like it does in the DM (Figure 4 b)). To enable effective cross-comparison, clusters are appropriately labelled in both the DM and updated p-chart (UPC), and their boundaries are denoted with the horizontal and vertical dashed purple lines, respectively.

The $C_{1}$ cluster consists of APA time window observations containing either no defective interval at all (542/961 observations), or have them appearing sporadically. The majority of the $C_{1}$ cluster defective intervals have APA time windows that occur fall below the CL of the UPC, indicating that they might be caused by spurious ventilatory sensor readings. Intermittent presence of defective intervals is also apparent in cluster $C_{2}$. However, this time they appear in much greater proportion compared to $C_{1}$ with the majority of observations falling above the $\mathrm{CL}$, of which 72 observations exhibited special cause variation (appeared above the $U C L$ ). This behaviour can best be explained by the influence of external factors on mechanical ventilation, the most likely being clinicians making changes in the modes of mechanical ventilation (support vs. control).

Cluster $C_{3}$ consists of a high proportion of APA time window observations with defective intervals which appear to form two distinctive patterns, best described by the sub-clusters $C_{3}^{1}$ and $C_{3}^{2}$ (segregated by dashed orange lines in DM and UPC). Cluster $C_{3}^{1}$ contains 129 observations, of which 77 consist of defective intervals only while the remaining ones were speckled with sporadic occurrence of non-defective intervals. While this clearly alludes to falsely triggered alerts, the same cannot be said for the sub-cluster $C_{3}^{2}$. Here we have a situation in which APA time window observations consist of a block of non-defective intervals followed by a block of defective ones hence implying that in these instances a clinical 
intervention transpired which resulted in the patient's health stabilisation (generally between 60 and 180 minutes since triggering the alert).

\section{Figure 4.}

Accuracy assessment of the VILIAlert system using a combination of the DM and p-chart: a) A DM provides an insight into patients data in the 6 hour long interval following an alert generation. APA time window observations are arranged based on the defective intervals occurrence patterns using AHC (Ward's method with Euclidean distances). Black and grey cells depict defective and non-defective intervals, respectively; b) A p-chart displaying the proportion of defective intervals per APA time window observation ordered in the same manner as in the DM.

\section{Discussion}

A lack of adequate metrics is a major barrier to effective performance analytics of real-time Clinical Decision Support Systems (CDSSs) where there are no indicators or tools available to identify whether an observation is genuine or not when triggering alerts. Traditional performance evaluation metrics (e.g. the area under the curve (AUC), precision/recall, etc), are unsuitable for this task as there is no mechanism which can help in distinguishing genuine from clinically insignificant alerts. The manual depiction of such information is complicated and time consuming requiring the expertise of the clinicians, hence the need for the development of new automated methods. The aim of this paper is to overcome the limitations of existing performance evaluation metrics by introducing the new DM-P approach which is based on the hybridisation of two techniques: DM (Defects Map) which is introduced in this paper for the first time and P-charts which are the type of SPC charts most often used in the field of quality control for analysing and monitoring dichotomous processes. We have demonstrated the use of the technique on the VILIAlert case study, by analysing patients' TV data recorded in a 6 hour long time interval that follows the alert generation.

DM relies on AHC (Ward's method with Euclidian Distances) to reorder APA time windows based on the similarity of their Dls occurrence patterns, and enables the p-chart creation using imposed data ordering. The P-chart is used then to estimate whether or not the 
triggered alert is genuine or not depending on whether the proportion of alerts' Dls recorded during the APA time window is within its CLs or not.

The combination of DMs and P-Charts have several advantages as reported by Al-Hussein (2009) who conclude that p-charts can act as a useful visual tool in a clinical setting. Firstly, p-charts become much more readable and interpretable, which is clearly evident by comparing Figures 3 and $4 \mathrm{~b}$ ) in which p-charts are constructed in chronological order compared to being ordered according to the DM's shuffled ordering of observations, respectively. Secondly, by having the observations that appear in the same order, enables us to perform effective cross-analysis between the p-chart and DM, and hence get both high and low-level insight into the nature of each generated alert which is beneficial from both clinical and algorithm development perspective. In other words, DM-P chart can be used for development of the interactive dashboards as the part of the nudge based intervention to increase the compliance with the best LPV practices, in the similar manner as it was previously done by Bourdeaux et al. [2].

Despite the criticism stating that using $\mathrm{AHC}$ for clustering binary valued data should be avoided [39], in their study Henry et al. [40] rebut these claims. Using Ward's method with Euclidean distances, these authors not only provided the evidence that AHC performs equally well when compared to the k-means clustering and Latent Class Analysis methods, but it also outperforms these two methods when clustering binary datasets with 50 observations or less which is common to many qualitative studies. On the other hand, progress of computationally efficient implementations, such as those based on reciprocal nearest neighbours algorithm [41], enabled Ward's method to be used for clustering datasets consisted of up to ca. $500 \mathrm{~K}$ observations [34]. This makes DM extremely versatile and an invaluable tool which can be used for assisting p-charts in performing SPC of dichotomous datasets of any size.

A motivation behind DM's invention is that the p-charts' CLs rely on the binomial distribution and one of the key assumptions for their construction is independence of events and their constant probability of occurrence [42]. In our study, the latter assumption may not be valid 
in cases in which there was an influence of external factors on mechanical ventilation during the APA time windows. These external factors may include, but they are not limited to: changing the mode of mechanical ventilation (control vs support), patient's behaviour (coughing, agitation, pain, etc) or clinical intervention (nebulisation, turning patient, suctioning, etc). Violation of this assumption may lead to an incorrect interpretation of the pcharts because their control limits will be either too wide or too narrow, regardless of the process' stability. To avoid misinterpretation in detecting special causes of variation, the literature recommends displaying p-charts alongside the xmr-charts [42]. However, in their study Poots and Woodcock [43] demonstrated that this approach leads to ambiguity when applied to data without an inherent ordering, which appears to be our use case. DM are invented with the purpose to accommodate this issue, as there was no viable alternative that could be used as the replacement for the xmr-charts.

The DM-P approach, introduced in this paper, is the sequel of our earlier work [13] in which we proposed shared frailty survival models in order to understand the behaviour of the realtime alerting CDSSs, as well as for the new algorithm's development and their cross comparison. The applicability of this technique was demonstrated using the same VILIAlert case study which we used in this paper. Speaking in the terms of survival analysis, the DI was the event of interest and our aim was to predict the position of appearance of the first interval (out of 24 available) in the APA time window that is going to be marked as defective. Although the longer times to first DI event occurrence (i.e. longer survival times) imply better performance of the alerting algorithm, they also indicate poorer ventilatory treatments in which the patients are exposed to injurious ventilation for a prolonged time period and thus increasing the risk of them getting VILI. The obtained results showed that in the VILIAlert study female patients received much poorer treatments than the males having a median time to occurrence of the first DI interval almost three times longer than the males ( 300 minutes vs 105 minutes respectively). The best shared frailty model which had the lognormal baseline function and the patient height as the covariate indicated that the shorter patients were also affected by the poorer treatments because the survival probability was reduced by 
a factor of 0.058 for each centimetre increment in height. The potential issue with survival analysis is the fact that it is slightly too conservative since, conservative an approach, since the time to appearance of the first DI block in the APA time window is taken as the survival time which might lead to underestimation of the CDSSs performance.

The DM-P approach, introduced here, is intended to complement the survival analysis approach as it provides clear insight into the distribution of DIs during the APA time windows. Using the p-chart we discovered that 338/1450 alerts had unusually high proportion of Dls per APA time window (i.e. more than 12/24 Dls) while the remaining alerts (1112/1450) were marked as genuine. Using DM (Figure 4 a)) we investigated that of that number only $129 / 338$ alerts were false (sub-cluster $C_{3}^{1}$ ), while the other instances can be classified as genuine because further inspection revealed that the unusually high number of defective intervals occurred due to the influence of external factors such as changing the mode of mechanical ventilation (cluster $C_{2}$ ) or clinical intervention (sub-cluster $C_{3}^{2}$ ). Given that the DMs are powered by $\mathrm{AHC}$, we need to mention that the resulting clustering solution could vary depending on the observations' order if tied distances or similarities exist in the input dataset or occur among updated clusters during joining phase [44]. This is also one of the main causes for misclustering of some observations. In our study, prior to performing $\mathrm{AHC}$, the dataset was arranged by the timestamps so that the defective intervals are placed at the end, as this approach produced the most meaningful results. Given that some APA time window observations were misclustered, as seen in clusters $C_{2}$ and $C_{3}$, we are inclined to report that the overall accuracy of the VILIAlert system is in the range of $76.7-91.1 \%$. This is significantly higher than what is reported by Herasevich et al. [15] whose CDDS scored a positive predictive rate of $59 \%$. During their study period, 111 alerts were triggered by 80 (out of 1159) ventilated patients, each manually inspected for genuine or not cases by the respiratory therapist who confirmed VILI risk in 65 cases in total.

\section{Conclusions and Future Directions}


In this paper, we introduced the DM-P approach for assessing the alerting performance of real-time clinical decision support systems in cases when there is no available information that could suggest whether the triggered alert is genuine or not. This approach successfully combines statistical process control charts (or more specifically p-charts) with the technique we introduced in this paper for the first time and called it DM. Effectiveness of the DM-P approach was demonstrated by analysing the performance of VILIAlert system which was designed to improve quality of ventilation by continuously monitoring mechanically ventilated patients in the ICU and to use these to send the alerts to clinicians if it predicts that configured ventilatory settings may lead to VILI.

Performance of any real-time alerting system depends on the quality of sensors' readings and the same applies for the VILIAlert system. By observing the distribution of Dls in cluster $C_{1}$ (Figure $\left.4 \mathrm{a}\right)$ ) and their proportion per APA time window, we can see that there is room for improvement of the mathematical kernel algorithm. Therefore, in our future work we will modify the mathematical kernel to apply some of the robust online filtering techniques (such as robust linear regression [45] or weighted running median [46] filters) to the calculated TVMLKG metrics (eq. 1), in order to reduce the negative impact of spurious ventilatory readings on the VILIAlert accuracy. Our intention is to use the DM-P approach to evaluate and compare the performances of these mathematical kernels and select the one which provides the optimal performance. We also plan to release the DM-P code in the form of open source R and Python packages, which will benefit the wider scientific community.

In this paper, we have demonstrated the usage of DM and p-charts in the assessment of the genuine or not cases of triggered alerts by using patient's tidal volume data in the period of 6 hours after they were triggered. However, we would like to highlight that, in the similar manner, DM can be used in conjunction with not just $\mathrm{p}$-charts but also with the other types of SPC charts such as c-harts, np-charts or u-charts, for monitoring other patients' physiological parameters (e.g. heart rate, oxygen saturation, etc.), which will also be the part of our future research. 


\section{References}

[1] P. J. Pronovost, "Enhancing physicians' use of clinical guidelines," JAMA, vol. 310, no. 23, pp. 2501-2502, 2013.

[2] C. P. Bourdeaux and et al., "Increasing compliance with low tidal volume ventilation in the ICU with two nudge-based interventions: evaluation through intervention timeseries analysis," BMJ Open, vol. 6, p. e010129, 2016.

[3] S. Eslami, N. F. De Keizer, A. Abu-Hanna, E. De Jonge and M. J. Schultz, "Effect of clinical decision support system on adherence to a lower tidal volume mechanical ventilation strategy," Journal of Critical Care, vol. 24, pp. 523-529, 2009.

[4] A. S. Slutsky and V. M. Ranieri, "Ventilator-Induced Lung Injury," The New England Journal of Medicine, vol. 369, pp. 2126-2136, 2013.

[5] A. Serpa Neto, "Lung-protective ventilation in intensive care unit and operation room: Tidal volume size, level of positive end-expiratory pressure and driving pressure (PhD Thesis)," Faculty of Medicine, University of Amsterdam, 2017.

[6] C. Putensen, J. Z. J. Theuerkauf, H. Wrigge and P. Pelosi, "Meta-analysis: ventilation strategies and outcomes of the acute respiratory distress syndrome and acute lug injury," Annals of Internal Medicine, vol. 151, no. 8, pp. 566-576, 2009.

[7] M. B. P. Amato and et al., "Effect of a protective-ventilation strategy on mortality in the acute respiratory distress syndrome," The New England Journal of Medicine, vol. 338, pp. 347-354, 1998.

[8] M. P. Young and et al., "Ventilation of patients with acute lung injury and acute respiratory distress syndrome: has new evidence changed clinical practice?," Critical 
Care Medicine, vol. 32, no. 6, pp. 1260-1265, 2004.

[9] C. R. Weinert, "Impact of randomised trial results on acute lung injury ventilator therapy in teaching hospitals," American Journal of Respiratory and Critical Care Medicine, vol. 167, no. 10, pp. 1304-1309, 2003.

[10] R. Kalhan and et al., "Underuse of lung protective ventilation: analysis of potential factors to explain physician behavior," Critical Care Medicine, vol. 34, no. 2, pp. 300306, 2006.

[11] W. Checkley, R. Brower, A. Korpak and B. T. Thompson, "Effects of a clinical trial on mechanical ventilation practices in patients with acute lung injury," American Journal of Respiratory and Critical Care Medicine, vol. 177, no. 11, pp. 1215-1222, 2008.

[12] A. J. Walkey and R. S. Wiener, "Risk Factors for Underuse of Lung Protective Ventilation in Acute Lung Injury," Critical Care, vol. 27, no. 3, pp. 323.e1-323.e9, 2012.

[13] A. H. Marshall and A. Novakovic, "Analysing the performance of a real-time healthcare 4.0 systems using shared frailty time to event models," in The 32nd IEEE International Symposium on Computer-Based Medical Systems (IEEE CBMS2019), Cordoba, Spain, 2019.

[14] C. J. Gillan, A. Novakovic, A. H. Marshall, M. Shyamsundar and D. Nikolopoulos, "Expediting assessments of database performance for streams of respiratory parameters," Computers in Biology and Medicine, vol. 100, pp. 186-195, 2018.

[15] V. Herasevich, M. Tsapenko, M. Kojicic, A. Ahmed, K. Rachul, C. Venkata, K. Shahjehan, S. J. Thakur, B. W. Pickering, J. Zhang, R. Hubmayr and O. Gajic, "Limiting ventilatorinduced lung injury through individual electronic medical record surveillance," Critical Care Medicine, vol. 39, no. 1, pp. 34-39, 2011. 
[16] K. Plathong and B. Surakratanasakul, "A study of integration Internet of Things with health level 7 protocol for real-time healthcare monitoring by using cloud computing," in The 2017 Biomedical Engineering International Conference (BMEiCON-2017), Hokkaido, Japan, 2017.

[17] Health Level Seven International, "About HL7," [Online]. Available: https://bit.ly/2tkavDk. [Accessed 11 April 2019].

[18] J. Zhang, W. Xu, J. Guo and S. Gao, "A temporal model in Electronic Health Record Search," Knowledge-Based Systems, vol. 126, pp. 56-67, 2017.

[19] F. Amato, G. De Pietro, M. Esposito and N. Mazzocca, "An integrated framework for securing semi-structural health records," Knowledge-Based Systems, vol. 79, pp. 99117, 2015.

[20] B. J. Devine, "Gentamicin therapy," Drug Intelligence \& Clinical Pharmacy, vol. 8, pp. 650-655, 1974.

[21] A. Duclos, N. Viorin, S. Touzet, P. Soardo, A.-m. Schott, C. Colin, J.-L. Peix and J.-C. Lifante, "Crude versus case-mix-adjusted control charts for safety monitoring in thyroid surgery," Quality \& Safety in Health Care, vol. 19, p. e17, 2010.

[22] R. v. B. Hollesen, R. L. R. J. Johansen, C. Rorbie, L. Munk, P. Barker and A. KjaerbyeThygesen, "Successfully reducing newborn asphyxia in the labour unit in a large academic medical centre: a quality improvement project using statistical process control," BMJ Quality \& Safety, vol. 27, pp. 633-642, 2018.

[23] M. Sadeghi, J. A. Leis, C. Laflamme, D. Sparkes, W. Ditrani, A. Watamaniuk, R. Taggar, F. Jinnah, M. Avaness, M. Vearncombe and A. B. Nathens, "Standardisation of perioperative urinary catheter use to reduce postsurgical urinary tract infection: an 
interrupted time series study," BMJ Quality \& Safety, Vols. doi: 10.1136/bmjqs-2017007458, 2018.

[24] M. A. Zafar, R. J. Panos, J. Ko, L. C. Otten, A. Gentene, M. Guido, K. Clark, C. Lee, J. Robertson and E. A. Alessandrini, "Reliable adherence to a COPD care bundle mitigates system-level failures and reduces COPD readmissions: a system redesign using improvement science," BMJ Quality \& Safety, vol. 26, pp. 908-918, 2017.

[25] J. C. Benneyan, "Use and interpretation of statistical quality control charts," International Journal for Quality in Health Care, vol. 10, no. 1, pp. 69-73, 1998.

[26] W. V. Padula, M. K. Mishra, C. D. Weaver, T. Yilmaz and M. E. Splaine, "Building information for systematic improvement of the prevention of hospital-acquired pressure ulcers with statistical process control charts and regression," BMJ Quality \& Safety, vol. 21, pp. 473-480, 2012.

[27] L. P. Provost and S. Murray, The Health Care Data Guide: Learning from Data for Improvement, San Francisco: Jossey-Bass, 2011.

[28] M. Templ, A. Alfons and P. Filzmoser, "Exploring incomplete data using visualization techniques," Advances in Data Analysis and Classification, vol. 6, no. 1, pp. 29-47, 2012.

[29] K. Li, Z. Ma, D. Robinson and J. Ma, "Identification of typical building daily electricity usage profiles using Gaussian mixture model based clustering and hierarchical clustering," Applied Energy, vol. 231, pp. 331-342, 2018.

[30] Z. Ma, R. Yan and N. Nord, "A variation focused cluster analysis strategy to identify typical daily heating load profiles of higher education buildings," Energy, vol. 134, pp. 90-102, 2017. 
[31] J. H. Ward, "Hierarchical grouping to optimize an objective function," Journal of the American Statistical Association, vol. 58, no. 301, pp. 236-244, 1963.

[32] R. K. Blashfield, "Mixture model tests of cluster analysis: Accuracy of four agglomerative hierarchical methods," Psychological Bulletin, vol. 83, no. 3, pp. 377-388, 1976.

[33] F. Murtagh, "Expected-time complexity results for hierarchic clustering algorithms which use cluster centres," Information Processing Letters, vol. 16, no. 5, pp. 237-241, 1983.

[34] T. Varin, R. Bureau, C. Mueller and P. Willett, "Clustering files of chemical structures using the Székely-Rizzo generalization of Ward's method," Journal of Molecular Graphics and Modelling, vol. 28, no. 2, pp. 187-195, 2009.

[35] R. Rosenthal and M. R. DiMatteo, "Meta-Analysis: Recent Developments in Quantitative Methods for Literature Reviews," Annual Review of Psychology, vol. 52, pp. 59-82, 2001.

[36] H. Akoglu, "User's guide to correlation coefficients," Turkish Journal of Emergency Medicine, vol. 18, no. 3, pp. 91-93, 2018.

[37] Y. H. Chan, "Biostatistics 104: Correlational Analysis," Singapore Medical Journal, vol. 44, no. 12, pp. 614-619, 2003.

[38] R Core Team, "R: A language and environment for statistical computing," R Foundation for Statistical Computing, 2018. [Online]. Available: https://www.R-project.org/.

[39] IBM Support Portal, "Clustering Binary Data (should be avoided)," [Online]. Available: https://ibm.co/2SGgNIT. [Accessed 11 October 2018].

[40] D. Henry, A. B. Dymnicki, N. Mohatt, J. Allen and J. G. Kelly, "Clustering methods with 
qualitative data: a mixed methods approach for prevention research with small samples," Prevention Science, vol. 16, no. 7, pp. 1007-1016, 2015.

[41] F. Murtagh, Multidimensional Clustering Algorithms, Vienna: Physica Verlag, 1985.

[42] M. A. Mohammed and P. Worthington, "Why traditional statistical process control charts for attribute data should be viewed alongside an xmr-chart," BMJ Quality \& Safety, vol. 22, pp. 263-269, 2013.

[43] A. J. Poots and T. Woodcock, "Statistical process control for data without inherent order," BMC Medical Informatics and Decision Making, vol. 12, p. 86, 2012.

[44] IBM Support Portal, "Hierarchical Cluster Analysis," [Online]. Available: https://ibm.co/2EWfkeE. [Accessed 211 2018].

[45] U. Gather, K. Schettlinger and R. Fried, "Online Signal Extraction by Robust Linear Regression," Computational Statistics, vol. 21, no. 1, pp. 33-51, 2006.

[46] K. Schettlinger, R. Fried and U. Gather, "Robust Filters for Intensive Care Monitoring Beyond the Running Median," Biomedizinische Technik, vol. 51, no. 2, pp. 49-56, 2006.

\section{Footnotes}

\section{Abbreviations:}

1. $\mathrm{AHC}-$ Agglomerative Hierarchical Clustering

2. APA Time Window- Analysed Post Alert Time Window

3. CDSS - Clinical Decision Support System

4. $\mathrm{CL}-$ Centreline

5. DI - Defective Interval

6. DM - Defects Map

7. EMR - Electronic Medical Record 
8. IBW - Ideal Body Weight

9. ICU - Intensive Care Unit

10. LCL - Lower Control Limit

11. LPV - Lung Protective Ventilation

12. MV - Mechanical Ventilator

13. SPC - Statistical Process Control

14. TV - Tidal Volume in $\mathrm{ml} /$ inspiration units

15. TVMLKG - Tidal Volume in $\mathrm{ml} / \mathrm{kg}$ units

16. UCL - Upper Control Limit

17. UPC - Updated P-Chart

18. VILI - Ventilator Induced Lung Injury

Funding: This research has been funded by Queen's University Belfast

Acknowledgement: The authors wish to acknowledge the Royal Victoria Hospital for facilitating the testing and validation of the VILIAlert system. We would also like to thank $\mathrm{Dr}$ Charles J Gillan (Institute of Electronics, Communications and Information Technology) and Dr Murali Shyamsundar (The Centre for Experimental Medicine, School of Medicine Dentistry and Biological Sciences) from Queen's University Belfast.

Competing interests: The authors have no competing interests

Ethics approval: This work was carried out as part of an ongoing Quality Improvement Project to improve the quality of mechanical ventilation in the Regional Intensive Care Unit, Royal Victoria Hospital, Belfast. Routinely collected anonymised data is used to develop the VILIAlert system and the ethical approval was deemed unnecessary by the research governance department of the Belfast Health and Social Care Trust.

Data sharing statement: The anonymised data set for this study is held securely at Queen's University Belfast. Data sharing regulations prevent this data from being made available publicly.

\section{[ End Document ]}


Table 1: Comparison of ventilated patients' demographics; triggered vs. not triggered alerts

\begin{tabular}{lccc}
\hline & \multicolumn{2}{c}{ Alert } & \multirow{2}{*}{ Univariate statistics } \\
\cline { 2 - 3 } & Triggered & $\begin{array}{c}\text { Not } \\
\text { Triggered }\end{array}$ & \\
\hline $\begin{array}{l}\text { Demographics } \\
\text { Gender distribution } \\
\text { Male, N (\%) }\end{array}$ & $298(55.49 \%)$ & $239(44.51 \%)$ & \\
$\quad$ Female, N (\%) & $262(77.51 \%)$ & $76(22.49 \%)$ & \\
Height in cm, MD & & & \\
(IQR) & & & \\
$\quad$ Overall & $165(14.8)$ & $170(15.0)$ & $W=119960 ; p<.0001 ; r=.3$ \\
Male & $170(13.0)$ & $175(10.0)$ & $W=41606 ; p<.001 ; r=.145$ \\
$\quad$ Female & $160(10.0)$ & $165(9.0)$ & $W=14717 ; p<.0001 ; r=.348$ \\
IBW in kg, MD (IQR) & & & \\
$\quad$ Overall & $59.2(15.4)$ & $65.9(13.6)$ & $W=120460 ; p<.0001 ; r=.304$ \\
Male & $65.9(11.8)$ & $70.5(9.1)$ & $W=41606 ; p<.001 ; r=.145$ \\
Female & $52.4(9.1)$ & $56.9(8.1)$ & $W=14717 ; p<.0001 ; r=.348$ \\
\hline
\end{tabular}




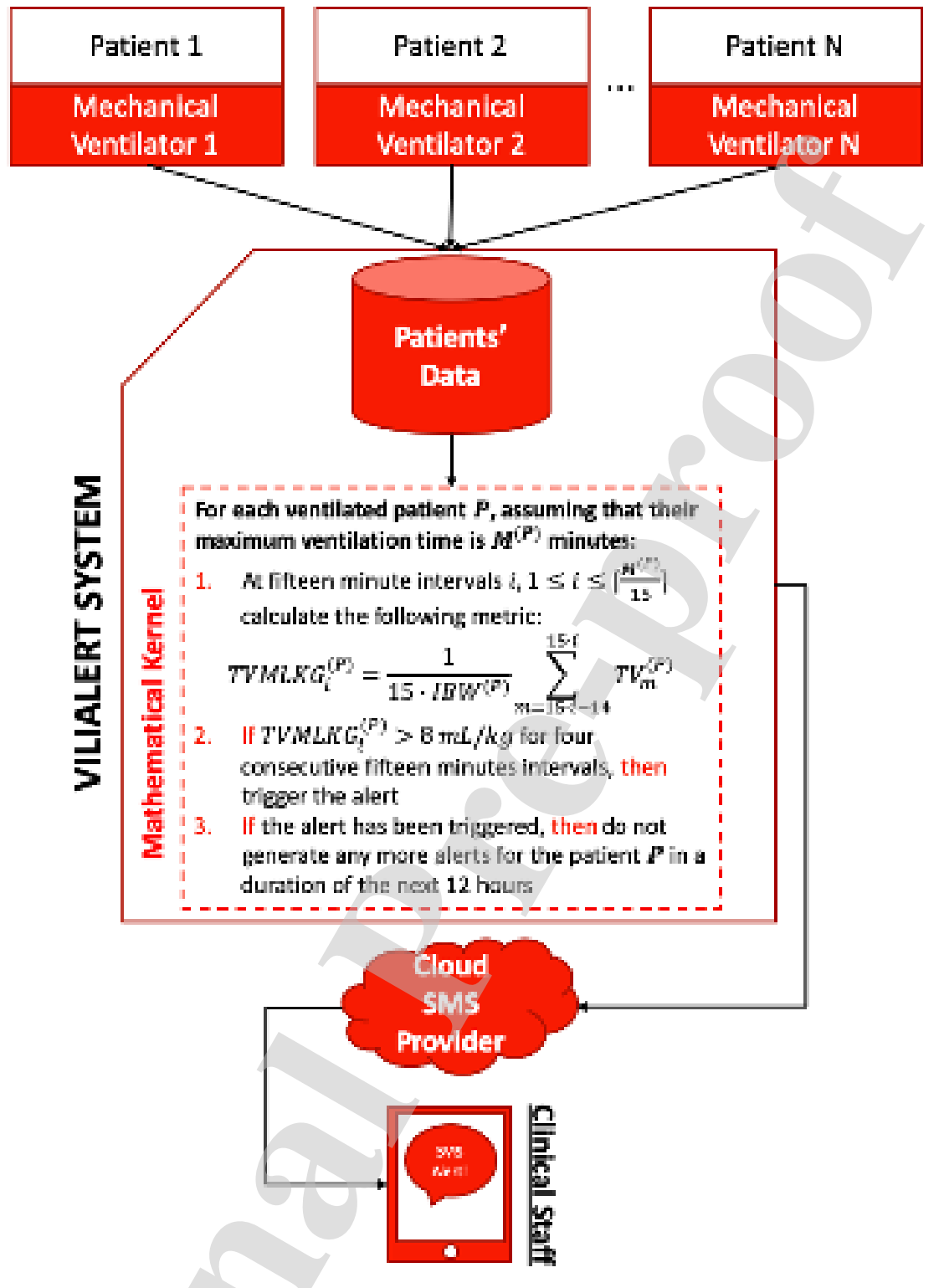

Figure 1. An architectural overview of the VILIAlert system. 


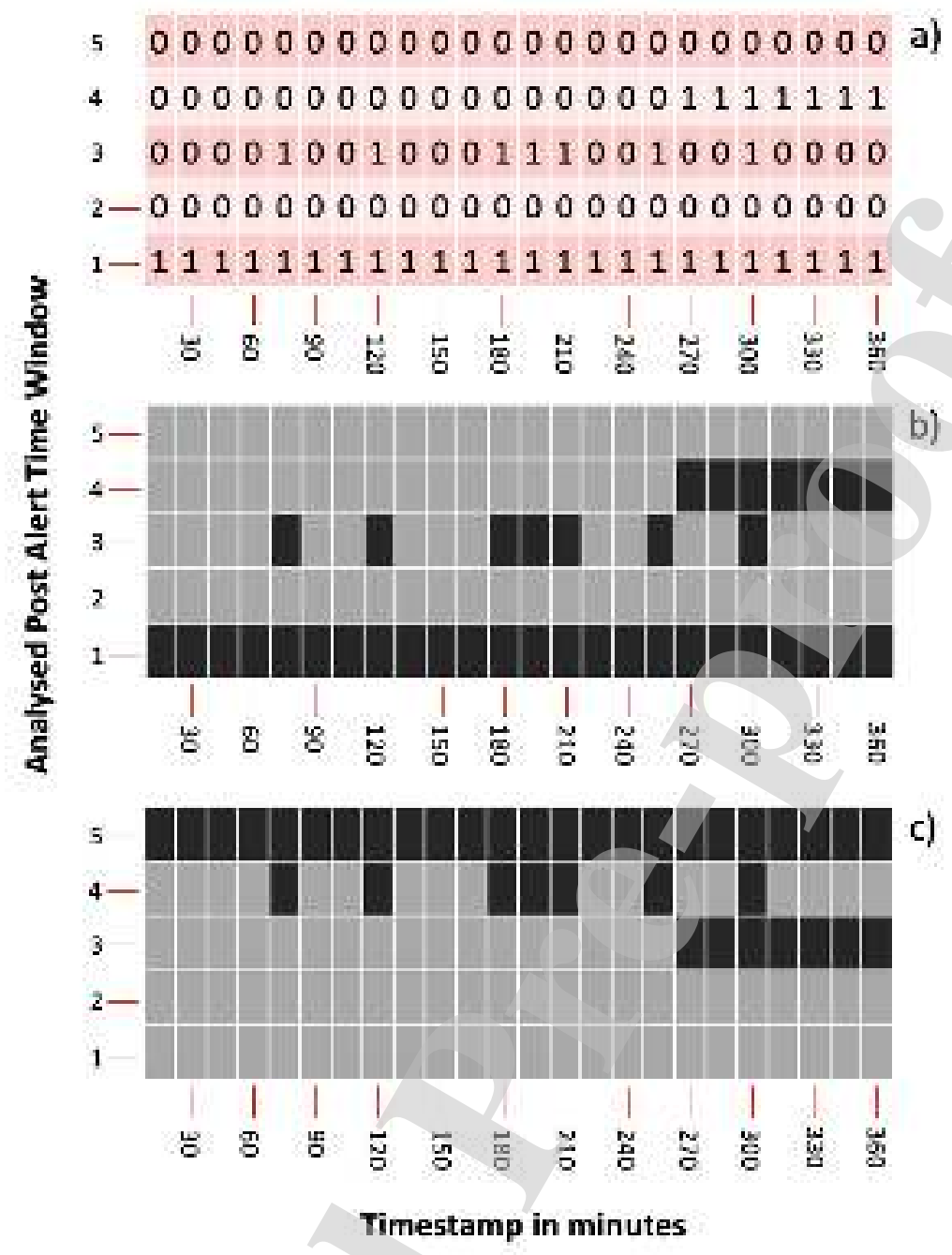

Figure 2. An illustration on how the DM is constructed: a) Theoretical representation of the data-matrix similar to the one used in our analysis. Symbols 1 and 0 denote defective and non-defective intervals, respectively; b) Visualisation of the previously introduced data-matrix using matrix plot visualisation technique. Defective and non-defective intervals are represented as the black and grey cells respectively; c) a DM representation of the datamatrix in which the APA time window observations are arranged based on defective intervals occurrence patterns using the AHC algorithm (Ward's method with Euclidean distances). 


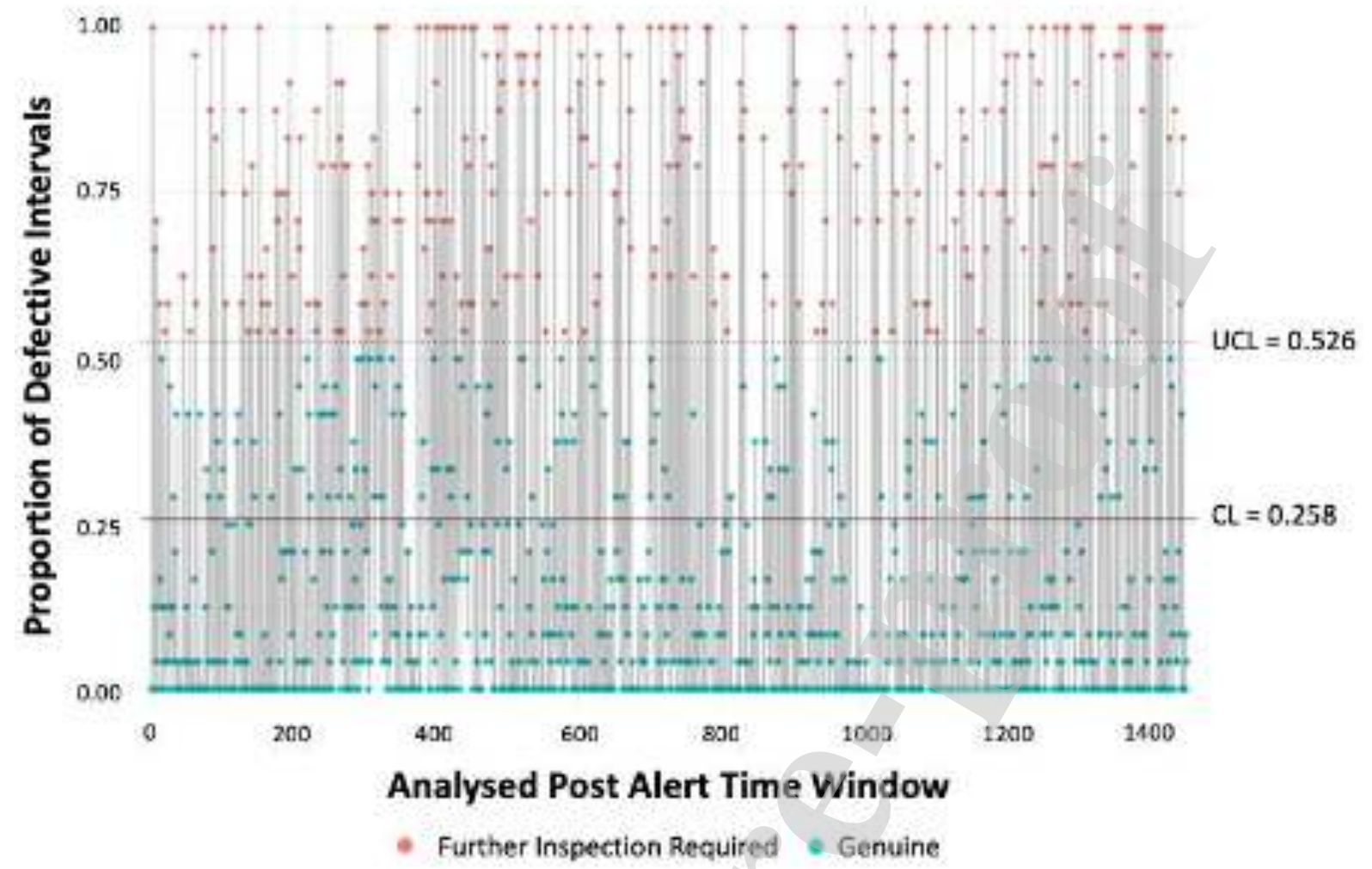

Figure 3. P-chart displaying the proportion of defective intervals relative to the APA time window observations which are arranged in chronological order. Upper control limit and centreline are denoted with dotted and solid black horizontal lines. Lower control limit is not set, as the $0 \%$ of defective intervals per APA time window is preferred. Light blue and red dots, depicts APA time window observations that indicate that the corresponding alert is either genuine or requires further investigation so that the authenticity of observations can be determined. 

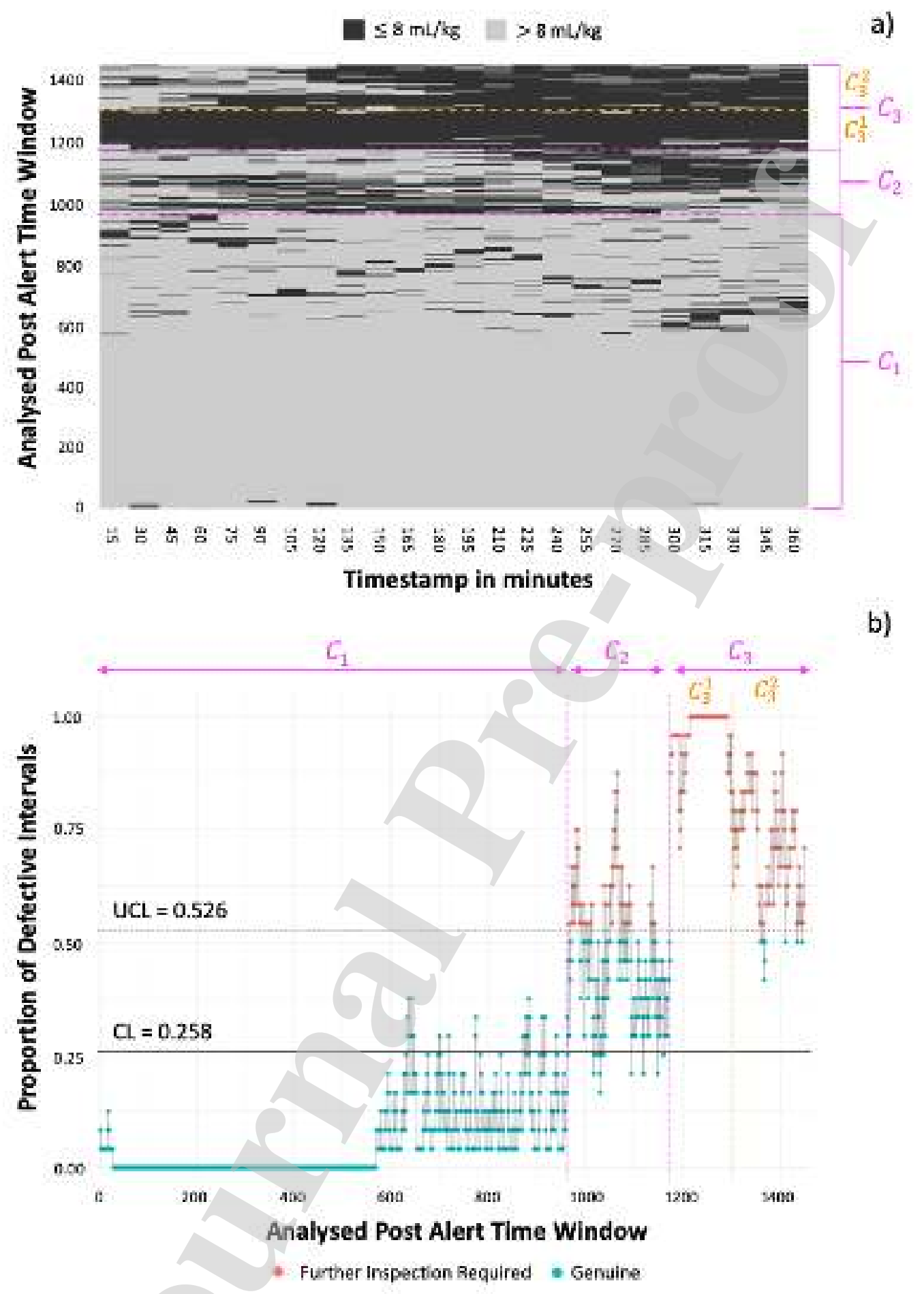

b)

Figure 4. Accuracy assessment of the VILIAlert system using a combination of the DM and p-chart: a) A DM provides an insight into patients data in the 6 hour long interval following an alert generation. APA time window observations are arranged based on the defective intervals occurrence patterns using AHC (Ward's method with Euclidean distances). Black and grey cells depict defective and non-defective intervals, respectively; b) A p-chart displaying the proportion of defective intervals per APA time window observation ordered in the same manner as in the DM. 


\section{Credit Author Statement}

Novakovic Aleksandar: Conceptualization, Methodology, Software, WritingReviewing and Editing.

Marshall Adele: Methodology, QI, Writing- Reviewing and Editing, 


\section{AUTHOR DECLARATION TEMPLATE}

We wish to confirm that there are no known conflicts of interest associated with this publication and there has been no significant financial support for this work that could have influenced its outcome.

We confirm that the manuscript has been read and approved by all named authors and that there are no other persons who satisfied the criteria for authorship but are not listed. We further confirm that the order of authors listed in the manuscript has been approved by all of us.

We confirm that we have given due consideration to the protection of intellectual property associated with this work and that there are no impediments to publication, including the timing of publication, with respect to intellectual property. In so doing we confirm that we have followed the regulations of our institutions concerning intellectual property.

We further confirm that any aspect of the work covered in this manuscript that has involved either experimental animals or human patients has been conducted with the ethical approval of all relevant bodies and that such approvals are acknowledged within the manuscript.

We understand that the Corresponding Author is the sole contact for the Editorial process (including Editorial Manager and direct communications with the office). $\mathrm{He} / \mathrm{she}$ is responsible for communicating with the other authors about progress, submissions of revisions and final approval of proofs. We confirm that we have provided a current, correct email address which is accessible by the Corresponding Author and which has been configured to accept email from a.h.marshall@qub.ac.uk

Signed by all authors as follows:

Professor Adele H Marshall

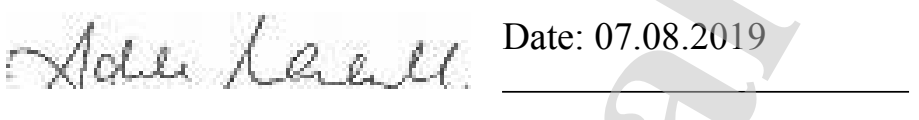

Dr Aleksandar Novakovic

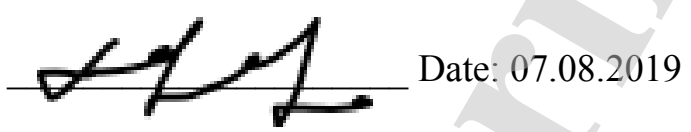

